developed and used to train the instructors with satisfied outcomes.

\section{ECONOMIC IMPACT AND CARE-SEEKING PATTERNS OF INJURIES IN BANGLADESH}

${ }^{1} Y$ Natalia Alfonso, 'David Bishai, ${ }^{2}$ Olakunle Alonge, ${ }^{3}$ Emdadul Hoque. ${ }^{1}$ Johns Hopkins University Bloomberg School of Public Health, Department of Family Population and Reproductive Health. Baltimore MD USA, 2Johns Hopkins University Bloomberg School of Public Health, Department of International Health. Baltimore MD USA, 3/nternational Centre for Diarrhoeal Disease Research, Bangladesh

10.1136/injuryprev-2016-042156.679

This study aims to provide an understanding of the economic hardship of individuals with unintentional injuries and economic recovery options in rural Bangladesh by assessing the variation in mortality and morbidity due to injuries and estimating the economic burden of injuries by type of injury.

Data were obtained from an annual demographic and injury surveillance system conducted in 7 sub-districts in rural Bangladesh during fiscal year 2014-2015. We tabulated injury prevalence and care-seeking patterns by injury type, age group and socioeconomic status (SES) and applied Chi square tests. A two part model of spending applied a generalised linear model to estimate the probability of any spending and amount of out-ofpocket costs per injury type. Lastly, a Markov model was developed to estimate the probability and cost for each type of injury.

There were 1,163,290 individuals and 119,669 self-reported injuries. The most common injuries were from falls (38\%), cuts (22\%), blunt objects (10\%), and transport (9\%). Drownings and violence injuries were more common among low SES, while electrocution were more common among high SES. Most injuries $(88 \%)$ sought treatment, $81 \%$ used village doctors, $3 \%$ were hospitalised for a median of 5 days, and $25 \%$ of the hospitalised had surgery. Of those treated, $4 \%$ reported no improvement in health. The mean and median cost for treated injuries, in 2015 BDT, was $\$ 1,302$ and $\$ 250$, respectively. Most treatments incurred expenditures on medicines (95\% median \$250), 31\% on transport cost (median $\$ 100$ ) and $15 \%$ on consultation fees (median \$220). The most expensive injury treatments were other $(\$ 6,125)$, attempted suicide $(\$ 2,000)$, violence $(\$ 680)$, and unintentional poisoning $(\$ 600)$. Other results will be shown later.

Data highlights injuries common among lowest or highest SES, treatment outcome patterns, and most common and most expensive health care services and injury types. This new evidence can improve understanding on health care use, the economic hardship and recovery options of individuals with injuries in rural Bangladesh.

\section{Posters Tuesday 20.9.20167}

\section{Brain Injuries}

\section{Post Tue 2.7}

\section{MULTI-FUNCTIONAL DRUG APPROACH FOR THE TREATMENT OF BRAIN INJURY}

${ }^{1}$ Adrian Harel, ${ }^{2}$ Mårten Kvist. ${ }^{1}$ Medicortex Finland Oy, Finland; ${ }^{2}$ University of Turku, Finland

10.1136/injuryprev-2016-042156.680
Background Traumatic brain injury (TBI) is one of the main causes of mortality among military personnel, children, young adults and athletes. Medicortex Finland has adopted a novel approach to attenuate secondary damages related to traumatic brain injury and stroke. TBI is manifested by early events and delayed secondary alterations. The latter include: mitochondrial dysfunction, lipid degradation and peroxidation and blood-brain barrier (BBB) disruption. This is followed by raised intracellular calcium influx and activation of proteases, resulting in axonal swelling, disconnection and degeneration. Pro-inflammatory factors are produced and secreted by local and infiltrated immune system cells, promoting the development of the inflammatory process. This series of events results in various neurological deficits. Since the degenerative process is mediated by multiple biological reactions, agents that target a single pathway are ineffective.

Method Medicortex presents a novel family of new chemical entities that cross the BBB, each possessing a penetrating head with a chemical spacer and two or more of the following properties: binding of free metal ions, anti-oxidation, anti-inflammation, and/or anti-bacterial. The lead compounds will be selected according to their solubility, stability and toxicity. In vitro and in vivo studies are conducted in order to explore the efficacy of the molecules as neuroprotective agents under different insults and to attenuate neural damage, utilising animal models of cortical impact brain injury.

Results The first compound, TBI-466, was tested by repeated injection at different concentrations and was found to be safe.

Conclusions Taken together, Medicortex's multi-functional drug agents will target biochemical pathways occurring at different time points post-injury, thereby attenuating and even preventing secondary TBI-associated neurological dysfunction and neuronal cell death.

\section{EPIDEMIOLOGY OF TRAUMATIC BRAIN INJURIES BASED ON HOSPITAL REPORTS IN METROPOLITAN FRANCE: WHICH ICD10 CODES SHOULD BE SELECTED?}

${ }^{1}$ Bertrand Thélot, ${ }^{1}$ Anne Pasquereau, ${ }^{1}$ Gaëlle Pedrono, 'Linda Lasbeur, ${ }^{2}$ Claire Jourdan, ${ }^{3}$ Hippolyte Kouadio, ${ }^{3}$ Emmanuel Rusch. ${ }^{1}$ Santé Publique France, Saint-Maurice, France; ${ }^{2}$ University Hospital, Montpellier, France; ${ }^{3}$ University Hospital, Tours, France

\subsection{6/injuryprev-2016-042156.681}

Background Traumatic brain injuries (TBI) result most often from injuries which could have been avoided through preventive measures. They have very costly human and financial consequences. To contribute to the epidemiological surveillance of TBI, the objective of this project was to analyse hospitalizations for TBI in France.

Methods Each hospitalisation results in a report containing information on the diagnosis, the treatment, the health condition upon discharge, etc. This database, called the Programme for the Medicalization of Information Systems (PMSI), is comprehensive. The main hospital diagnosis (MD) and associated diagnosis (AD) are coded in the International Classification of Diseases, $10^{\text {th }}$ Revision (ICD10). The selections were made from different ICD10 code lists used in the literature: "Intracranial injury" (S06 codes) always selected for TBI analysis; "Fracture of vault of skull, etc." (S02.0, S02.1, S02.3, S07.1) often retained (OR); "Fracture of skull and facial bones, etc." (S02.7, S02.8, S02.9, S07.0, S07.8, S07.9, S09.7, S09.8, S09.9) sometimes retained (SR). The selection must be made on all diagnoses (MD and AD), since the TBI can be coded as an $\mathrm{AD}$ in case of multiple injuries. 
Results In 2013, there were 134,546 hospitalizations with at least one diagnosis coded as S06, 8,292 additional hospitalizations coded as OR, and 6,821 hospitalizations coded as SR, representing a total of 149,659 hospitalizations. The fact of adding to the selection of S06 codes hospitalizations with OR codes or hospitalizations with OR and SR codes results in an increasing number of hospitalizations: $6.2 \%$ and $11.2 \%$ respectively.

Conclusions The analysis of hospitalizations for TBI should not be limited to $\mathrm{S} 06$ codes because they produce too many false negatives. The analysis of false positives and negatives associated with the addition of OR or OR + SR codes requires the use of a gold standard to conclude on the best selection and estimate hospital morbidity due to TBI.

\section{NOVEL USE OF ELECTRONIC HEALTH RECORDS TO ADVANCE RESEARCH AND MANAGEMENT OF PAEDIATRIC CONCUSSIONS}

${ }^{1}$ Kristy B Arbogast, ${ }^{1}$ Allison E Curry, ${ }^{2}$ Juliet Haarbauer-Krupa, ${ }^{1}$ Kristina B Metzger, ${ }^{1}$ Melissa R Pfeiffer, ${ }^{3}$ Mark R Zonfrillo, ${ }^{2}$ Matthew J Breiding, ${ }^{1}$ Ronnie Kessler, 'Julia Vanni, ${ }^{2}$ Jeneita Bell, ${ }^{1}$ Christina L Master. 'The Children's Hospital of Philadelphia (CHOP), Philadelphia, Pennsylvania, USA; ${ }^{2}$ Centers for Disease Control and Prevention (CDC), Atlanta, Georgia, USA; ${ }^{3}$ Brown University, Providence, Rhode Island, USA

\subsection{6/injuryprev-2016-042156.682}

Background While our understanding of concussion as an important public health issue among children has grown, broad description of paediatric concussions is limited to high school sports or those treated in an emergency department (ED). Further, nonspecialist providers report inadequate training and infrastructure to systematically diagnose and manage these patients. To address these gaps, CHOP and CDC have initiated a novel collaborative effort to examine whether electronic health records (EHRs) can expand traumatic brain injury (TBI) surveillance and researchwith a focus on concussion-and to assess whether EHR-based initiatives can improve TBI management.

Methods All patients, age 0-17 years, with at least one clinical encounter with an ICD-9-CM diagnosis of concussion in the CHOP EHR system (7/2010-6/2014) were selected and their initial concussion-related encounter identified.

Results 14,054 patients were included (average age: 12.1 years). Initial analyses indicate that: $50 \%$ of patients had their first encounter within primary care and $27 \%$ within specialty care; half of all concussions among 5-11 year olds were sports/recreation-related; and introduction of a concussion clinical support tool substantially improved documentation of concussion-specific assessment in the EHR (2\% before vs. $70 \%$ after implementation in July 2012).

Conclusions This collaborative program leverages the strength of a linked EHR system throughout a large healthcare network $(>1$ million annual visits) to provide a comprehensive system-wide assessment of paediatric concussion across the developmental age spectrum beyond the ED setting. This is the first such US assessment that included a diverse demographic and socioeconomic sample. This work highlights the potential of EHRs to guide clinical management and facilitate research that can lead to improved concussion prevention and diagnosis.

\section{CONCUSSION EDUCATION AND THE THEORY OF PLANNED BEHAVIOUR}

Lindsay Sullivan, Michal Molcho. Department of Health Promotion, School of Health Sciences, NUI Galway, Galway, Ireland

\subsection{6/injuryprev-2016-042156.683}

Background Sports-related concussion among youth has been identified as a public health problem. As a result, an abundance of concussion education programmes for youth athletes have been developed. Although some of these programmes have resulted in an increase in concussion knowledge, these programmes have failed to increase athletes' concussion reporting behaviours. Accordingly, research suggests a need for theorydriven concussion interventions that go beyond those that aim at increasing concussion knowledge alone. This study will apply the theory of planned behaviour (TPB) to the development and evaluation of a targeted concussion education programme for athletes aged 13 to 18 years old. The primary aim of this study is to assess the immediate and short-term impact of the programme on intention to report concussion and concussion reporting behaviours; and to explore, the process of implementation and the relationship between this process and the programme's outcomes.

Methods This study will employ a cluster randomised control trial, with data collection at baseline (T0), immediately post-intervention (T1), and three-months after programme implementation (T2). The sample will be comprised of county-level Gaelic Athletic Association (GAA) clubs throughout the Republic of Ireland and will include teams with athletes aged 13 to 18 years old. Using a range of structured questionnaires and qualitative measures, data will be captured on athletes' (i) attitudes towards concussion and concussion reporting, (ii) subjective norms, (iii) perceived behavioural control, (iv) intention to report concussion, (v) concussion reporting behaviour and (vi) programme fidelity.

Results Preliminary results from the evaluation and issues with programme implementation will be presented. Quantitative data will be analysed with SPSS Version 22. Thematic analysis will be used to analyse qualitative data.

Conclusions Findings from this evaluation will be used to assess the efficacy of the TPB in programme development and evaluation and will support the knowledge and practice of primary and secondary concussion prevention. Results from the process evaluation will assist in helping understand the context within which the programme was implemented and how these characteristics may affect the quality of implementation.

\section{COACH COMMUNICATION ABOUT CONCUSSION SAFETY AND THEIR PERCEIVED ABILITY TO ASSESS AND MANAGE CONCUSSION}

Lindsay Sullivan, Michal Molcho. Department of Health Promotion, School of Health Sciences, NUI Galway, Galway, Ireland

\subsection{6/injuryprev-2016-042156.684}

Background Annually, it is estimated that 3.8 million sports- and recreation-related concussions are sustained in the USA alone, with a particularly high incidence among adolescents. At youth sporting events, however, medical professionals are rarely 